\title{
Non-invasive parameters for the detection of variceal bleed in patients of liver cirrhosis, an experience of a tertiary care hospital in Pakistan
}

\author{
Anam Umar', Fakhar Ali Qazi', Rukhsana Abdul Sattar', Beena Umar ${ }^{2}$ \\ ${ }^{1}$ Department of Medicine, Jinnah Postgraduate Medical Centre, ${ }^{2}$ Department of Histopathology and Microbiology, Aga Khan University \\ Hospital, Karachi, Pakistan
}

\section{A B S T R A C T}

Objective: Cirrhotic patients commonly undergo screening endoscopy for the existence of esophageal varices. The use of this invasive procedure which is expensive, poorly tolerable and generally not acceptable for the patients is increasing due to increasing number of patients with chronic liver disease and their enriched survival. In this study, our aim is to identify clinical, biochemical, and ultrasonography parameters which might noninvasively predict the presence of esophageal varices and risk of bleeding in patients with liver cirrhosis. Material and Methods: Total 150 Patientsof chronic liver disease admitted in ward-5, JPMC(Sep 2011-Feb 2012) with a complaint of hematemesis or melena were included in the study. Platelet counts of 75,000 to $150,000 / \mu \mathrm{L}$ was defined as grade 1 thrombocytopenia, 50,000 to $<75,000 / \mu \mathrm{L}$ as grade II, 25,000 to $<50,000 / \mu \mathrm{L}$ as grade III and below $25,000 / \mu \mathrm{L}$ as grade IV. The normal range for the INR is $0.8-1.2$. Portal vein size of $1.2 \mathrm{~cm}$ or above was taken as dilated. Spleen of $>13 \mathrm{~cm}$ was considered as enlarged in our study. Results: Out of 72 patients of variceal bleed 69 (46\%) were males and $81(54 \%)$ were females. Thrombocytopenia was present in 64 (88\%) patients with mean platelet count of $85.86 / \mu \mathrm{L}( \pm 69.79)$. Deranged coagulation profile was present in $56(77 \%)$ cases with mean INR of $1.63( \pm 0.5)$. Portal vein diameter (PVD) of $>1.2 \mathrm{~cm}$ was found in $46(63.8 \%)$ of patients with mean PVD of $1.22( \pm 0.3023)$ and splenic size of $>13 \mathrm{~cm}$ was reported in $54(75 \%)$ cases with mean splenic diameter of $14.5 \mathrm{~cm}( \pm 2.39)$. Conclusion: Thrombocytopenia, deranged coagulation profile, large splenic size, and dilated portal vein strongly predict the risk of variceal bleeding.

Key words: Variceal Bleed, Precipitating factors, Cirrhosis of the liver, Outcome

\section{INTRODUCTION}

In underdeveloped countries, liver cirrhosis is a major cause of morbidity and mortality owing to unawareness of the patients, inadequate facilities and financial implications associated with the disease. In western world chronic alcohol consumption account for majority of the cases but in our part of the world infections by hepatotropic viruses (Hepatitis B \& C) are most likely responsible. Liver cirrhosis follows an indolent course and eventually patients succumb to the complications of liver decompensation characterized by variceal bleeding from portal hypertension, ascites, hepatorenal syndrome, hepatic encephalopathy and spontaneous bacterial peritonitis. ${ }^{1}$ Esophageal varices are generally the most common clinical manifestation of portal hypertension and ruptured esophageal varices are a dreaded complication of portal hypertension, accounting for approximately 3200 deaths annually attributed to cirrhosis. ${ }^{2}$ Varices usually form when hepatic vein pressure gradient (determinant of portal pressure) exceeds $10 \mathrm{mmHg}$ and bleeds when it exceeds $12 \mathrm{mmHg}$. In cirrhosis, there is compression of hepatic veins by regenerating nodules along with collagen deposition in sinusoids and venules, factors responsible for increased intrahepatic resistance. The increase in blood flow is incompletely understood but is 
probably due to circulating vasodilators and alterations in fluid and electrolyte balance. All these interactions result in the development of increase portal pressure and Porto-systemic collateral formation. These collaterals decompress the portal circulation by shunting the blood to the heart via the systemic venous circulation and the major sites of these collaterals are the esophagus, rectum, umbilicus and retro peritoneum. ${ }^{3,45}$ Gastro esophageal varices are present in approximately $50 \%$ of patients with cirrhosis with a lifetime incidence of $90 \%$. Their presence also correlate with the severity of disease, being more common in Child Pugh Class C patients as compared to Child Pugh Class A patients ( $85 \%$ versus $40 \%)^{6,7,8}$ (Table 1). Variceal hemorrhage is a medical emergency. Approximately one third of patients with esophageal varices experience variceal bleeding with a mortality rate of up to $20 \%$. After an initial episode, there is $70 \%$ chance of re-bleeding within a year with a mortality rate of up to $33 \%$ in the absence of any treatment ${ }^{9}$ Due to this reason a screening endoscopy is indicated in all patients with newly diagnosed cirrhosis to minimize the complications and also to reduce mortality associated with acute variceal bleed. ${ }^{2}$ It has also been suggested to repeat endoscopy at every 2-3 years interval in patients without varices and at 1-2 years interval in patients with small varices to evaluate for progression ${ }^{10}$ Current treatment modalities have substantially reduced the rate of first and recurrent bleeding and has also decreased the mortality associated with acute variceal bleed. ${ }^{11}$ However screening all patients with endoscopy will significantly increase the cost and has also variable patient compliance. A number of other factors such as platelet count, coagulation profile, splenic size and portal vein diameter also correlate with the risk of variceal bleed and can be assessed by less invasive and routine tests carried out on the patients of cirrhosis. Due to cost effectiveness and to limit the number of endoscopic procedures, these parameters become more significant and carry paramount importance in making it possible for endoscopy to be carried out only in selected group of patients thus to avoid un-necessary intervention and at the same time not to miss patients at risk of bleeding. Therefore, the present study has been carried out to identify clinical, biochemical and ultrasonographic parameters which might non-invasively predict the existence and the risk of variceal bleed.

\begin{tabular}{lccc} 
Table 1: Child pugh classification \\
\hline & $\mathbf{1}$ & $\mathbf{2}$ & $\mathbf{3}$ \\
\hline Bilirubin $(\mathrm{mg} / \mathrm{dl})$ & $<2$ & $2-3$ & $>3$ \\
Serum albumin $(\mathrm{g} / \mathrm{dl})$ & $>3.5$ & $2.8-3.5$ & $<2.8$ \\
Grade of encephalopathy & None & Mild & Moderate to severe \\
Grade of ascites & None & Mild & Moderate to severe \\
INR & $<1.2$ & $1.2-1.7$ & $>1.7$ \\
\hline Child A: $5-6$, Child B: $7-9$, Child C: $10-15$ & &
\end{tabular}

\section{MATERIAL AND METHOD}

A total of 150 patients with chronic liver disease, admitted to the department of medicine, ward 5, Jinnah Postgraduate Medical Centre from September 2011 to February 2012 were included in this study. All patients were categorized into two groups according to the presence or absence of upper GI bleed in the form of hematemesis or melena. Diagnosis of liver cirrhosis was based on combination of clinical signs \& symptoms, laboratory findings (deranged coagulation profile, reversal of albumin/globulin ratio) and Ultrasonographic findings (size and echogenicity of the liver). Patients suffering from acute liver failure, non-cirrhotic portal hypertension, and tropical splenomegaly were excluded from the study.

A structured proforma was used for data collection. Detailed history was obtained and patients were examined with special attention to anemia, jaundice, palmarerythema, spider nevi, gynaecomastia, edema, splenomegaly and ascites. Relevant hematological, biochemical and radiological investigations were ordered including complete blood count, urea, creatinine and electrolytes, liver function tests, coagulation profile, serum proteins, serum albumin and globulin ratio, HBsAg, Anti HCV Antibody, urine $\mathrm{D} / \mathrm{R}$, ascetic fluid $\mathrm{D} / \mathrm{R}$, serum ammonia, chest X-Ray, ultrasound abdomen and upper GI endoscopy (as part of routine workup) (Table 2 ).

Upper gastrointestinal bleed was defined on the basis of hematemesis or melena. Thrombocytopenia as platelet count of $<150,000 / \mathrm{mm}^{3}$, deranged coagulation profile as INR of $>1.2$, splenomegaly as splenic size of $>13 \mathrm{~cm}$ anddilated portal vein diameter of $>1.2 \mathrm{~cm}$ on ultrasound. Platelet counts of 75,000 to $150,000 / \mu \mathrm{L}$ was defined as grade I thrombocytopenia, 50,000 to $75,000 / \mu \mathrm{L}$ as grade II, 25,000 to $50,000 / \mu \mathrm{L}$ as grade III, and below $25,000 / \mu \mathrm{L}$ as grade IV thrombocytopenia.

Statistical analysis was done using SPSS version 19 and the results were obtained in the terms of frequencies and percentages for descriptive analysis. Chi-Square and student $t$ tests were used to analyze the numerical data and $p$ value of $<0.05$ was set to be statistically significant.

\section{RESULTS}

\section{Age \& Gender}

There were eighty one $(n=81,54 \%)$ females and sixty nine $(n=69,46 \%)$ males. The range of patient's age was $14-83$ yrs. $58 \%(\mathrm{n}=87$ ) of the patients in our study belong to the age group of $40-60$ years with mean age of 50.9 years and Standard deviation of \pm 13.171 (Figures 1 \& 2). 


\begin{tabular}{|c|c|c|c|c|c|}
\hline & \multicolumn{2}{|c|}{ Without bleed } & \multicolumn{2}{|c|}{ With bleed } & \multirow[t]{2}{*}{ p-value } \\
\hline & Mean & Std. Deviation & Mean & Std. Deviation & \\
\hline Hemoglobin (mg/dl) & 8.429 & 2.2389 & 8.184 & 2.0675 & 0.492 \\
\hline Mean cell volume (fl) & 88.421 & 14.1289 & 91.584 & 10.0995 & 0.142 \\
\hline $\operatorname{TLC}\left(\times 10^{9} / \mathrm{L}\right)$ & 8.790 & 6.1854 & 8.453 & 5.7380 & 0.739 \\
\hline Platelets $/ \mathrm{mm}^{3}$ & 113.96 & 67.188 & 86.10 & 69.645 & $0.012^{*}$ \\
\hline Random blood sugar (mg/dl) & 137.58 & 84.530 & 137.64 & 68.215 & 0.997 \\
\hline Urea $(\mathrm{mg} / \mathrm{dl})$ & 57.09 & 47.601 & 52.11 & 35.582 & 0.492 \\
\hline Creatinine (mg/dl) & 1.4016 & 1.04940 & 1.1420 & 0.61934 & 0.084 \\
\hline Serum sodium (meq/l) & 132.472 & 7.1617 & 133.277 & 9.3108 & 0.578 \\
\hline Serum potassium (meq/l) & 4.2748 & 3.40198 & 3.9015 & 0.75980 & 0.400 \\
\hline Serum chloride (meq/l) & 102.746 & 8.4363 & 103.127 & 6.2709 & 0.792 \\
\hline Total bilirubin $(\mathrm{mg} / \mathrm{dl})$ & 4.2211 & 5.38458 & 3.3395 & 3.87788 & 0.300 \\
\hline Direct bilirubin (mg/dl) & 1.9992 & 3.25431 & 1.6781 & 2.40724 & 0.540 \\
\hline Indirect bilirubin (mg/dl) & 2.1063 & 2.44664 & 1.6953 & 1.72525 & 0.300 \\
\hline $\operatorname{ALT}(u / l)$ & 44.81 & 53.126 & 51.90 & 39.677 & 0.428 \\
\hline $\mathrm{AST}(\mathrm{u} / \mathrm{l})$ & 85.15 & 64.431 & 99.00 & 109.509 & 0.439 \\
\hline $\operatorname{ALP}(u / l)$ & 372.20 & 285.201 & 296.13 & 226.103 & 0.118 \\
\hline GGT (u/l) & 58.13 & 74.675 & 47.33 & 35.865 & 0.358 \\
\hline Total proteins (mg/dl) & 8.141 & 7.1363 & 8.202 & 9.6373 & 0.970 \\
\hline Serum albumin (mg/dl) & 3.125 & 0.5835 & 3.623 & 4.1489 & 0.376 \\
\hline Globulin (mg/dl) & 4.037 & 0.9202 & 3.677 & 0.8849 & 0.050 \\
\hline $\mathrm{A} / \mathrm{G}$ ratio & 0.810 & 0.1880 & 0.845 & 0.2007 & 0.390 \\
\hline INR & 1.3731 & 0.45532 & 1.6385 & 0.50358 & $0.001^{*}$ \\
\hline Serum ammonia $(\mu \mathrm{g} / \mathrm{dl})$ & 164.00 & 74.740 & 253.33 & 98.845 & $0.016^{*}$ \\
\hline Liver span (cm) & 11.562 & 2.1332 & 12.029 & 3.3295 & 0.584 \\
\hline Portal vein diameter $(\mathrm{cm})$ & 1.092 & 0.2583 & 1.224 & 0.3023 & $0.007^{*}$ \\
\hline Spleen size $(\mathrm{cm})$ & 13.082 & 2.3503 & 14.565 & 2.3994 & $0.000^{*}$ \\
\hline Splenic vein diameter $(\mathrm{cm})$ & 1.167 & 0.2066 & 0.700 & 0.2828 & $0.041^{*}$ \\
\hline
\end{tabular}

* Significant P-value

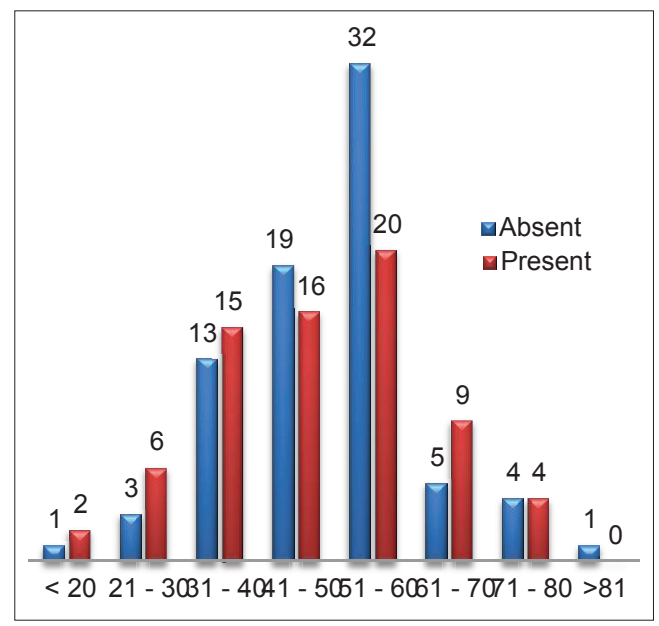

Figure 1: Age prevalence

\section{Frequency of viral hepatitis}

The most common cause of chronic liver disease was found to be HCV in our patients. Frequency of viral hepatitis as a cause of chronic liver disease is mentioned in Figure 3.

\section{Frequency of upper GI bleed}

72 patients in our study presented with GI Bleed that makes $48 \%$ of total cases while 78 patients $(52 \%)$ patients had no history of upper GI bleed. Out of 72 patients, $22(15 \%)$ patients presented with hematemesis, $12(8 \%)$ had melena

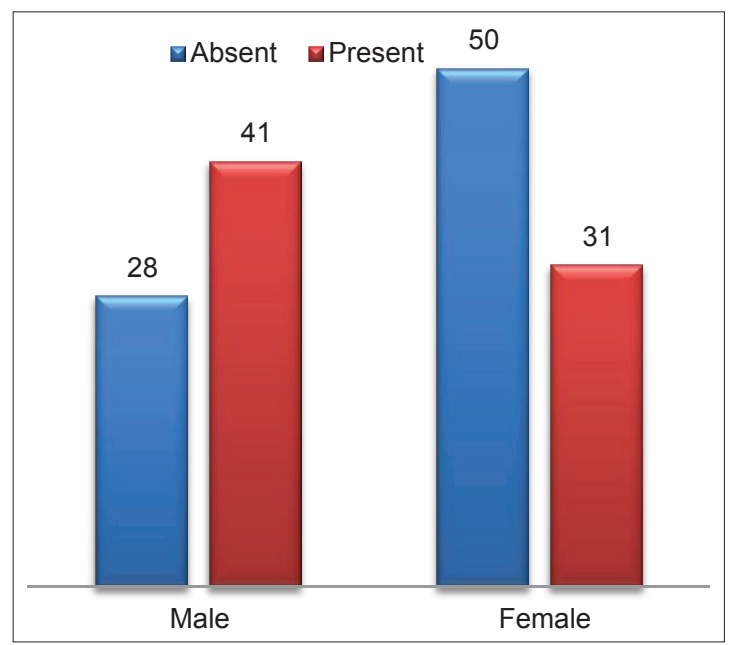

Figure 2: Gender prevalence

and $38(25 \%)$ patients presented with both hematemesis and melena (Figure 4).

\section{NON-INVASIVE PARAMETERS FOR ESOPHAGEAL VARICES}

\section{Thrombocytopenia}

Thrombocytopenia was present in $64(88 \%)$ patients, with mean platelet count of $85.86 / \mu \mathrm{L}( \pm 69.79)$ in 
patients with upper GI bleed. While 63 patients with no history of upper GI bleed had thrombocytopenia with mean platelet count of 113.96 (p-value 0.014). Statistically significant relationship was found between the severity of thrombocytopenia and risk of bleed in CLD patients (Figure 5) (Pearson's Chi Square: $\mathrm{p}$ value $=0.009)$.

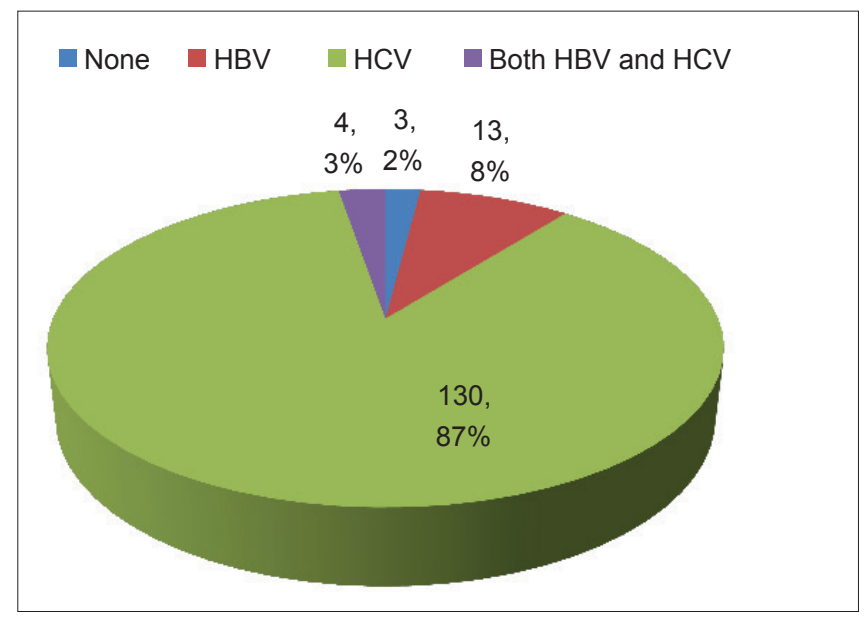

Figure 3: Frequency of viral hepatitis

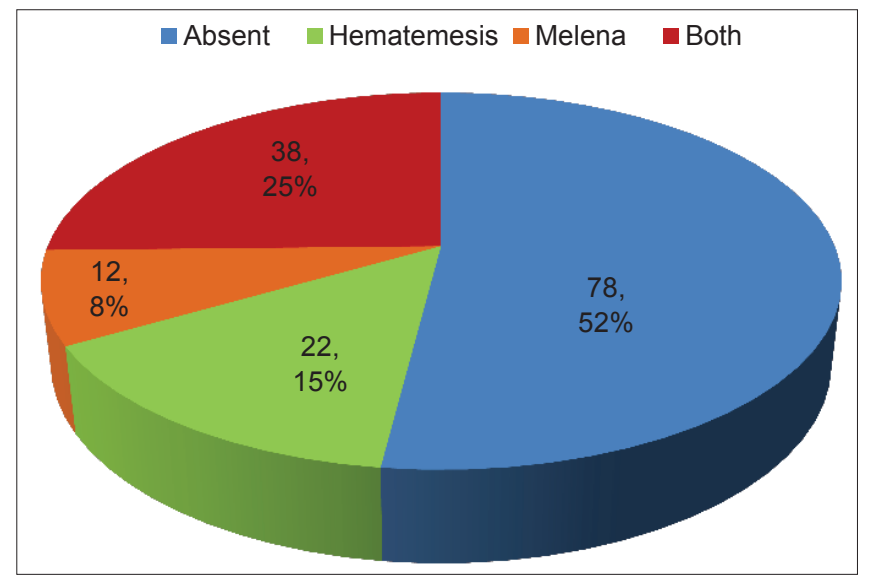

Figure 4: Frequency of upper GI bleed

\section{Coagulation profile}

Deranged coagulation profile was present in $56(77 \%)$ cases with GI Bleed, with mean INR of $1.63( \pm 0.5)$ in contrast to only 39 patients (50\%) without upper GI Bleed had deranged coagulation profile with a mean INR of $1.37( \pm 0.45)(\mathrm{p}$ value $=0.001)$. Level of significance was determined by application of Pearson chi square ( $\mathrm{p}$-value 0.001) (Figure 6).

\section{Portal vein diameter}

Portal vein diameter of $>1.2 \mathrm{~cm}$ was found in $46(63.8 \%)$ patients who presented with GI Bleed with mean PVD of $1.22 \mathrm{~cm}( \pm 0.3023),(\mathrm{p}$ value $=0.005)$ while $35(44.8 \%)$ patients without bleed had dilated portal vein on ultrasound with mean PVD of $1.092 \mathrm{~cm}( \pm 0.02)$. Level of significance was determined by application of Pearson chi square (p-value 0.022) (Figure 7).

\section{Splenic size}

A splenic size of $>13 \mathrm{~cm}$ was found in $54(75 \%)$ cases having mean splenic diameter of $14.5 \mathrm{~cm}( \pm 2.39)$ (p value $<0.001)$. On the other hand, 35 (44\%) patients without GI Bleed had splenomegaly with mean splenic diameter of $13.08 \mathrm{~cm}( \pm 2.35)$. Level of significance was determined by application of Pearson chi square (p-value 0.000) (Figure 8).

\section{DISCUSSION}

Acute Upper GI hemorrhage is a medical emergency with high morbidity and mortality, numerous hospital admissions and a high burden on health care providers. ${ }^{12,13}$ Esophageal varices are the major cause of upper GI hemorrhage in our setting as compared to peptic ulcer in western population. Despite significant improvements in early diagnosis and advancements made in the treatment modalities, the mortality rate of first variceal hemorrhage is still $20 \%$. Numerous studies have demonstrated the significance of pharmacologic therapy for primary prevention of

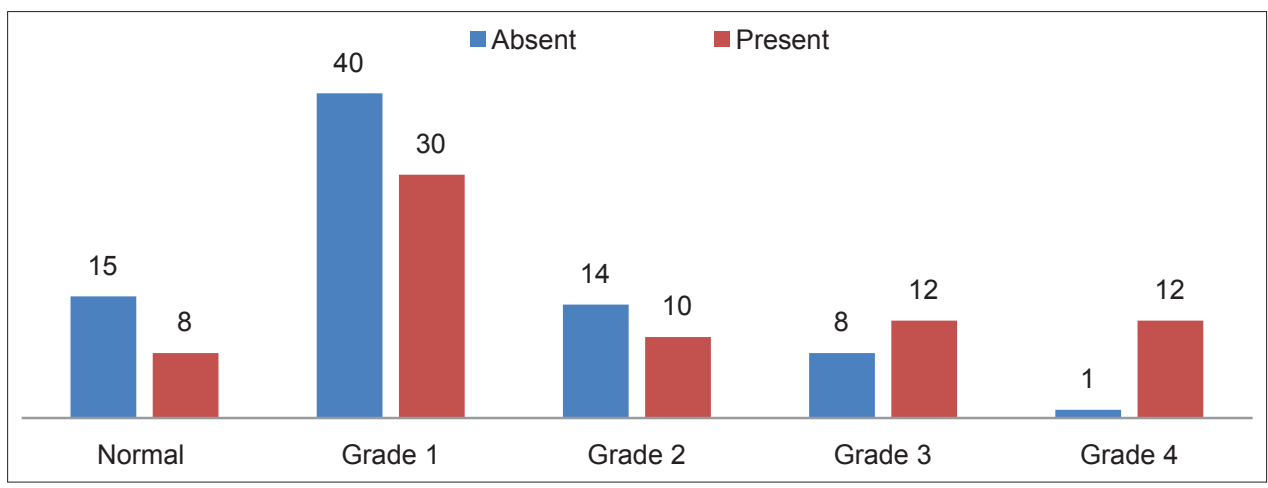

Figure 5: Grades of thrombocytopenia vs Gl bleed (statistically significant difference was found between the groups by applying Pearson Chi Square (p-value 0.005)) 


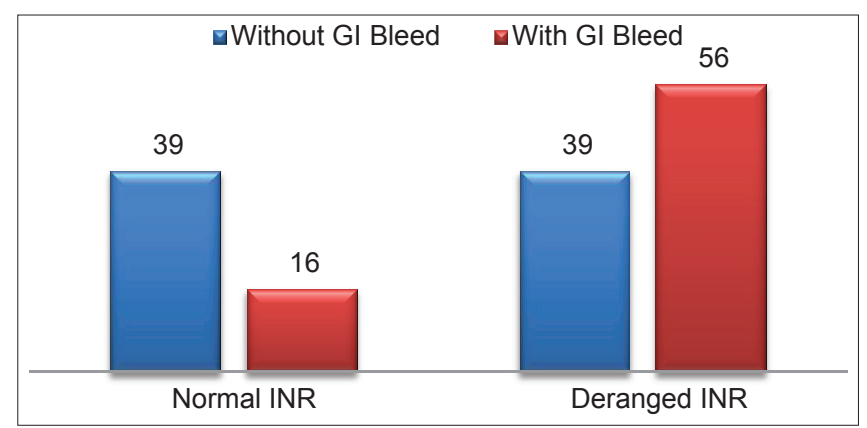

Figure 6: Coagulation profile vs GI bleed

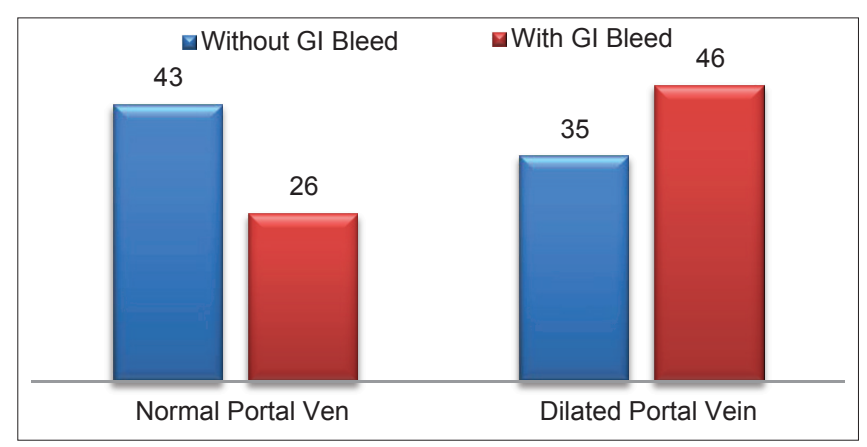

Figure 7: Portal vein size vs Gl bleed

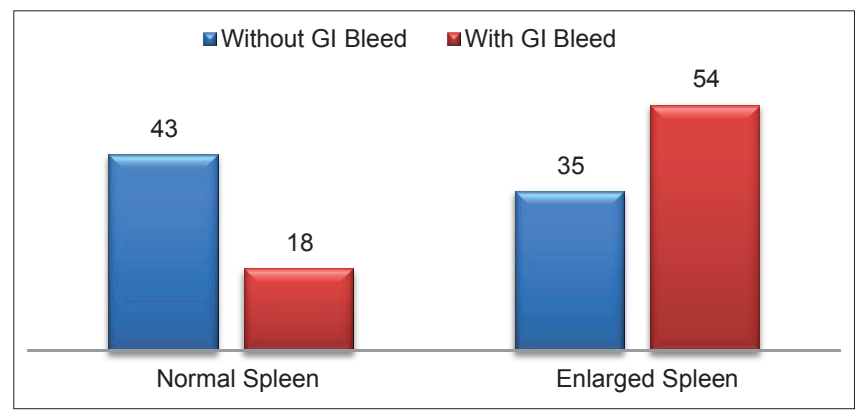

Figure 8: Splenomegaly vs GI bleed

variceal bleeding, emphasizing the importance of screening endoscopy in all newly diagnosed cirrhotic patients for the presence of varices. ${ }^{9,14}$ Therefore, there is a particular need for the identification of noninvasive parameters that strongly predict the presence of esophageal varices as that might decreases the medical, public and economic burden of the disease.

Our Study demonstrated that thrombocytopenia, deranged coagulation profile, presence of portal hypertension with splenomegaly and portal vein diameter are strong predictors of developing variceal bleed presenting clinically as upper GI hemorrhage.

Earlier, the pathophysiology of thrombocytopenia in liver disease was linked to the presence of hyperspleenism, but now it has been discovered that thrombopoetin production is dependent on functioning liver cell mass and is responsible for reduced thrombopoiesis and consequently peripheral thrombocytopenia in patients of advanced liver disease. ${ }^{15,16}$ Prolonged PT is also related to the severity of liver diseases and is also a simple, inexpensive and accurate marker of liver impairment and a predictor of upper gastrointestinal bleeding in chronic liver disease. ${ }^{17}$

Splenomegaly and portal vein diameter are also reliable predictors of variceal hemorrhage, in a study carried out by Mandal et $\mathrm{al}^{18}$ it has been shown that grading of esophageal varices also correlate with splenic size and portal vein diameter. In their study average portal vein diameter and splenic size in high grade varices were 14.43 $\pm 0.86 \mathrm{~mm}$ and $15.36 \pm 2.14 \mathrm{~cm}$. Sharma and Agarwal in their study had noted that clinically palpable spleen was associated with high grade varices however they did not measure the splenic size radiologically. ${ }^{19}$ The measurement of the splenic diameter using ultrasonography is easily obtainable, reproducible, non-invasive and is routinely performed on patients with cirrhosis. In another study carried it was demonstrated that the platelet count/ spleen diameter ratio is also useful in the discrimination of large versus small varices and in their study a platelet spleen diameter ratio of less than 909 was statistically significant in predicting large varices. ${ }^{20}$ Farooqi et al ${ }^{21}$ also found out that platelet count of $<65 \times 10^{3} / \mu \mathrm{L}$, serum albumin $<2.2 \mathrm{~g} / \mathrm{dl}$ and portal vein diameter of $>13 \mathrm{~mm}$ are independent and significant predictors of esophageal varices on endoscopy.

Though, upper GI endoscopy remains the gold standard for the diagnosis of esophageal varices in cirrhotic patients, from the present study and a number of other studies it has been found that certain non-endoscopic parameters such as thrombocytopenia $\left(<75,000 / \mathrm{mm}^{3}\right)$, coagulation profile (PT $>1.3)$, spleen size $(>13 \mathrm{~cm})$, and portal vein diameter $(>1.2 \mathrm{~cm})$ are significant and reliable predictors of esophageal varices on endoscopy, therefore screening endoscopy could be safely limited to the group of cirrhotic patients with no history of upper GI bleed but in the presence of above deranged parameters.

\section{REFERENCES}

1. Joel J, Heidelbaugh and Bruderly M. Cirrhosis and chronic liver failure Part 1. Diagnosis and Evaluation. Am Fam Physician 2006; 74(5):756-762.

2. Mohammad KT, Mohammad HS, Farhang $S$ and Jalilvand $M$. Portal hemodynamics as predictors of high risk esophageal varices in cirrhotic patients. World J Gastroenterol 2008; 14(12):1898-1912.

3. Toubia $\mathrm{N}$ and Sanyal AJ. Portal hypertension and variceal hemorrhage. Med Clin N Am 2008; 92:551-574.

4. Mahl TC and Groszmann RJ. Pathophysiology of portal 
hypertension and variceal bleeding. Surg Clin North Am 1990; 70(2): 251-266.

5. Kumar A, Sharma $P$ and Sarin SK. Hepatic venous pressure gradient measurement: time to learn! Indian J Gastroenterol 2008; 27(2):74-80.

6. Gracia-Taso G, Sanyal AJ, Grace ND and Carey W. Prevention and management of Gastroesophageal varices and variceal hemorrhage in cirrhosis. Hepatology 2007; 46(3):923-938.

7. Hong WD, Zhu QH, Huang ZM, Chen XR, Jiang ZC, Xu SH, et al. Predictors of esophageal varices in patients with HBV related cirrhosis: a retrospective study. BMC Gastroenterology 2009; 9(11):1-7.

8. Rye K, Scott R, Mortimore G, Lawson A, Andrew A and Freeman J. Towards noninvasive detection of Oesophageal varices. Int: J Hepatol 2012; 1:1-9.

9. Khaderi S and Barnes D. Preventing a first episode of esophageal variceal hemorrhage. Cleve Clin J Med 2008; 75(3):235-244.

10. Nashaat $E H$, Abd-Elaziz $H$, Sabry $M$ and Ibrahim AA. Non-endoscopic parameters of esophageal varices and portal hypertensive gastropathy. Nature and science 2010; 8(6):43-50.

11. Chen $\mathrm{YI}$ and Ghali P. Prevention and management of gastroesophageal varices in cirrhosis. Int J Hepatol 2012; 3:1-6.

12. Tsemeli NE, Kotsaftis PS, Savopoulos CG, Hatzitolios AL, Kaiafa GD and Kounanis AD. Incidence and etiology of acute non-malignant upper gastrointestinal bleeding in northern Greece. J Gastroenterol Hepatol 2007; 22(7):1009-1013.

13. Manning Dimmitt LL, Dimmit SG and Wilson GR. Diagnosis of gastrointestinal bleeding in adults. Am Fam Phys 2005; 71(7):1339-1346
14. Lowe RC. Pharmacological therapy for the treatment of esophageal varices. Minerva gastroenterol Dietol 2006; 52(2):215-223.

15. Peck-Radosavljevic M. Thrombocytopenia in liver disease. Can J gastroenterol 2000; 14:60-66.

16. Zaman A, Becker T, Lapidus $\mathrm{J}$ and Beener K. Risk Factors for the presence of varices in cirrhotic patients without a history of variceal hemorrhage. Arch Intern Med 2001; 161(21):2564-2570.

17. Siddiqui SA, Ahmed M, Ghani MH, Memon MA, Mustafa G and Ghori MA. Coagulation abnormalities in patients with chronic liver disease in Pakistan. J Pak Med Assoc 2011; 61(4):363-367.

18. Mandal L, Mandal SK, Bandyopadhyay D and Datta S. Correlation of portal vein diameter and splenic size with gastroesophageal varices in cirrhosis of liver. J Ind Aca Clin Med 2011; 12(4):266-270.

19. Sharma SK and Aggarwal R. Prediction of large esophageal varices in patients with cirrhosis of the liver using clinica laboratory and imaging parameters. J Gastroenterol Hepatol 2007; 22(11):1909-1915.

20. Saranagapani A, Shanmugam C, Kalyanasundaram M, Rangachari B, Thangavelu $\mathrm{P}$ and Subbarayan JK. Noninvasive prediction of large esophageal varices in chronic liver disease patients. Saudi J Gastroenterol 2010; 16(1): 38-42.

21. Farooqi $\mathrm{Jl}$, Ahmed $\mathrm{H}$, Ikramullah $\mathrm{Q}$, Ahmad $\mathrm{F}$ and Rehman $\mathrm{M}$. Predictors of esophageal varices in patients of liver cirrhosis. JPMI 2007; 21(1):60-64.

\section{Authors Contribution:}

$\mathrm{AU}$ and FAQ - designed the study, collected and analyzed the data and scripted the parts of manuscript; BU - scripted the manuscript and reviewend the results; RAS - reviewed the manuscript.

Source of Support: Nil, Conflict of Interest: None declared. 